\title{
Étude Rétrospective Des Atteintes Rénales Toxiques Medicamenteuses Au Centre Hospitalier Universitaire De Yaoundé (2009-2014)
}

\author{
Emilienne Grâce Nkoyock, Engineer - Epidemiologist, Msc In \\ Public Health \\ Efoulan District Hospital, Yaoundé-Cameroon \\ Blaise H. Nguendo Yongsi, PhD In Health Geography, \\ Epidemiologist, Senior Lecturer \\ IFORD-University of Yaoundé II, Cameroon \\ Justin Ndié, Inf. - Epidemiologist, Msc In Public Health \\ Ministry of Public Health, Cameroon \\ Patrice Emmanuel Awono Ateba, Inf. - Epidemiologist, Msc In \\ Public Health \\ Yaoundé General Hospital, Cameroon
}

doi: 10.19044/esj.2016.v12n27p149 URL:http://dx.doi.org/10.19044/esj.2016.v12n27p149

\begin{abstract}
Background: Renal drug toxicity are affections that attacking the renal parenchyma as a result of the consumption of drug substance. In Cameroon, anarchic consumption of medicinal drug subsequent of a growth in unauthorised sales drug increase the risk of developing these pathologies.

Objective: This study aims at describing and laying the epidemiological profile of drug-induced toxic renal diseases in Yaoundé University Teaching Hospital over the last 6 years $(2009-2014)$.

Materials and methods: A retrospective cross-sectional study was carried out from the $1^{\text {st }}$ of July to the $30^{\text {th }}$ of November 2015 , at the polyvalent reanimation service and the haemodialysis unit of the Yaoundé University Teaching Hospital. With the aid of a questionnaire, the files of patients consulted and hospitalised in these services over a period of 6 years $\left(1^{\text {st }}\right.$ January 2009 to $31^{\text {st }}$ December 2014) were examined. Included in this study, patients suffering from renal drug toxicity. The data input, treatment and analysis were carried out with the help of Epi Info 7 and SPSS 22 software.

Results: Forty-four (44) cases renal drug diseases representing $18.3 \%$ of the 240 files examined were noted. The average age of the patients was $48 \pm 18$ years and varied between 11 and 87 years. The male sex constituted the majority of the suffers cases with a sex ratio of $2.67(\mathrm{M} / \mathrm{F})$. Acute renal
\end{abstract}


infections with frequency of $65.9 \%$, were most dominant of drug affections. Traditions concoctions $(50 \%)$, gentamycin $(11.4 \%)$ and paracetamol $(9.1 \%)$ were the most incriminating drug in the occurrence of these renal drug affecting. The evolution was noted by a $43 \%$ complete recovery against a $14 \%$ death.

Conclusion: Renal drug toxicities represent a non-negligible proportion of the renal affection ailments in the specialised centres like Yaoundé University Teaching Hospital. The elaboration and putting into action a sensitization plan for populations on the proves necessary, as this would help improve pharmacovigilance and also reduce the consumption of illicit drugs to a greater extent responsible for renal problems.

Keywords: Epidemiological profile, drugs, renal drug toxicities, nephropathy, nephrotoxicity, CHUY

\section{Résumé}

Contexte : Les atteintes rénales toxiques médicamenteuses sont des affections touchant le parenchyme rénal inhérentes à la consommation des substances médicamenteuses. Au Cameroun, la consommation anarchique du médicament subséquente à l'expansion de la vente illicite, augmente le risque de survenue de ces pathologies.

Objectif : Cette étude vise à décrire et dresser le profil épidémiologique des atteintes rénales toxiques médicamenteuses au Centre Hospitalier Universitaire de Yaoundé-Cameroun (CHUY) au cours des six dernières années (2009 - 2014).

Matériels et méthodes : Une étude transversale rétrospective dans les services de réanimation polyvalente, de l'unité d'hémodialyse et de Médecine du CHUY a été réalisée du $1^{\text {er }}$ Juillet au 30 Novembre 2015. À l'aide d'un questionnaire, les dossiers des patients consultés et hospitalisés dans ces services sur une période de six ans ( $1^{\mathrm{er}}$ Janvier 2009 au 31 Décembre 2014) ont été compulsés. Étaient inclus dans cette étude, les malades souffrants d'atteintes rénales toxiques médicamenteuses. La saisie, le traitement et l'analyse des données ont été réalisées à l'aide des logiciels Epi Info 7 et SPSS 22.

Résultats : Quarante-quatre (44) cas d'atteintes rénales médicamenteuses représentant $18,3 \%$ des 240 dossiers compulsés ont été recensés. L’âge moyen des patients était de $48 \pm 18$ ans et variait entre 11 et 87 ans. Le sexe masculin était majoritairement atteint avec un sex-ratio $(\mathrm{H} / \mathrm{F})$ de 2,67 . Les infections rénales aigues (IRA) avec une fréquence de 65,9\%, étaient les affections médicamenteuses dominantes. Les décoctions traditionnelles $(50 \%)$, la gentamicine $(11,4 \%)$ et le paracétamol $(9,1 \%)$ ont été les médicaments les plus incriminés dans la survenue de ces atteintes rénales 
médicamenteuses. L'évolution a été marquée par $43 \%$ de guérison complète contre $14 \%$ de décès.

Conclusion : Les atteintes rénales toxiques médicamenteuses représentent une proportion non négligeable des affections rénales dans les centres spécialisés dont le CHUY. L'élaboration et la mise en œuvre d'un plan de sensibilisation des populations sur la consommation avisée des substances médicamenteuses au Cameroun s'avère nécessaire, car elle permettra d'améliorer la pharmacovigilance et de limiter la consommation des médicaments illicites en majeur partie, responsables des atteintes rénales.

Mots-clés : Profil épidémiologique, médicaments, atteintes rénales toxiques médicamenteuses, néphropathie, néphrotoxicité, CHUY

\section{Introduction}

Les atteintes rénales toxiques médicamenteuses sont des affections graves touchant le parenchyme rénal. Elles sont consécutives à la consommation des substances médicamenteuses. Plus de 1100 médicaments ont été référencés comme potentiellement toxiques (Frexinos et Buscail, 2003). En outre, les réactions médicamenteuses ont de graves répercussions sur les services de soins, et certains pays ne consacrent pas moins de 15 à $20 \%$ de leur budget de la santé aux problèmes liés aux médicaments (OMS, 2004).

Selon l'OMS (2008), 6,5\% des admissions dans les centres de soins et hôpitaux sont dues aux effets secondaires des médicaments. En plus, elle notait que les réactions indésirables aux médicaments dans certains pays se situaient entre le $4^{\text {ème }}$ et le $6^{\text {ème }}$ rang des causes de mortalité. En effet, les conséquences sont variées allant des déséquilibres électrolytiques, des fibroses interstitielles aux néphrites interstitielles chroniques, sans compter les cancers des voies urinaires (Dahl, 2001 ; Nortier, Depierreux et Vanherweghem, 1999). Par ailleurs, la prise d'anti-inflammatoires non stérö̈diens (AINS) en vente libre peut avoir de graves conséquences pour les personnes souffrant d'insuffisance rénale (Lord et Ménard, 2002a).

En Afrique, 35\% des cas d'insuffisances rénale aiguës (IRA) sont causés par les produits de l'ethnomédecine (Nortier et al., 1999). Toutefois, l'augmentation des patients dans les centres d'hémodialyse serait due à la consommation anarchique des faux médicaments achetés en majorité dans la rue (Fondjo, 2009). En effet, selon Auzanneau (2001) et Ebelle Lottin (2007) faisant suite à Youssif (2002), environ 46,9\% des populations consommaient les médicaments sans aucun avis médical.

$\mathrm{Au}$ Cameroun, la pharmacovigilance et le contrôle du circuit des médicaments restent une faiblesse majeure du système de santé. De fait, la circulation et la vente du médicament illicite ont libre cours prédisposant les populations à une automédication courante. Ce qui les expose ainsi aux 
multiples risques sanitaires dont la néphrotoxicité médicamenteuse. En effet, $50 \%$ de la population au Cameroun, pratiquent l'automédication sans avis médical (Ebelle Lottin, 2007). Selon Tanguy (2011) à la suite du Ministère de la Santé publique (2010), près de 40\% de médicaments vendus au Cameroun proviennent de la contrebande. En 2009, le circuit illicite à lui tout seul, représentait 20 à $25 \%$ du marché national du médicament. Pourtant, les conséquences de la consommation de ces produits sont variées à l'instar des résistances multiples, des accidents allergiques, des intoxications, des insuffisances rénales et hépatiques voire des décès (Mbassi-Bikele, 2014). Dans les centres spécialisés en général et au CHUY en particulier, très peu de travaux de recherche sur les conséquences des effets indésirables des médicaments sur le rein sont menés. Ainsi, la présente étude vise à dresser la situation des atteintes rénales toxiques médicamenteuses au CHU de Yaoundé à travers les données sociodémographiques, la typologie des atteintes et les médicaments incriminés.

\section{Méthodologie \\ Cadre de l'étude}

Notre étude s'est déroulée au CHU de Yaoundé. Il s'agit d'un hôpital de référence de première catégorie, crée en 1978. Il est situé dans le District de Santé de Biyem-Assi, Arrondissement de Yaoundé VI, Région du CentreCameroun.

\section{Matériels et méthodes}

Il s'agit d'une étude transversale rétrospective réalisée du $1^{\text {er }}$ Juillet au 30 Novembre 2015 (5mois) dans les services de réanimation polyvalente, de l'unité d'hémodialyse et de Médecine du CHUY. Étaient inclus dans cette étude, les malades souffrants d'atteintes rénales toxiques médicamenteuses. À l'aide d'un questionnaire, les dossiers de 240 patients consultés et hospitalisés dans ces services sur une période de six ans ( $1^{\mathrm{er}}$ Janvier 2009 au 31 Décembre 2014) ont été compulsés.

Les variables de cette étude étaient les données Sociodémographiques (sexe, âge, profession, lieu de résidence des patients), la typologie de l'atteinte (signes cliniques et biologiques, antécédent, motif de consultation, signes fonctionnels), les médicaments incriminés (classe médicamenteuse, traitement, l'évolution) et l'évolution du malade après traitement.

La saisie, le traitement et l'analyse des données ont été effectués à l'aide des logiciels Epi Info 7 et SPSS 22. Les analyses descriptives univariées ou tri à plat ont permis de caractériser les malades et les atteintes à travers les tendances centrales (moyenne ou médiane), les caractéristiques de dispersion (écart type) et la distribution de fréquence des modalités de notre variable d'intérêt. Ensuite, les analyses bivariées pour établir les liens entre 
les variables à l'aide du test de $\mathrm{Khi}^{2}$. Le seuil de significativité retenue était $\mathrm{p}<0,05$. Le test de $\mathrm{khi}^{2}$ ne donnant pas le sens de la relation entre les variables, le calcul du risque relatif (RR) et de l'intervalle de confiance (IC) à $95 \%$ a permis de compléter les analyses. Ainsi, la valeur du RR des IRA comme risque de référence avec comme hypothèse nulle $\left(\mathrm{H}_{0}\right)$ : les variables sont indépendantes et comme hypothèse alternative $\left(\mathrm{H}_{1}\right)$ : les variables sont liées.

\section{Résultats}

Des 240 dossiers de patients souffrant de néphropathies compulsés, 44 cas d'atteintes rénales toxiques médicamenteuses représentant 18,30\% des malades ont été recensés.

Le sex-ratio $(\mathrm{H} / \mathrm{F})$ était de 2,67. L'âge des patients variait de 11 à 87 ans avec un âge moyen de $48 \pm 18$ ans. Les tranches d'âge les plus affectées étaient respectivement celles de 45 à 54 ans et 65 ans et plus avec 20,45\% chacune. Les patients issus du secteur informel étaient les plus touchés avec une fréquence de $27 \%$ suivi des agriculteurs $(20,5 \%)$, en troisième position des fonctionnaires avec $15,9 \%$. Par contre, les moins touchés étaient les inactifs $(6,9 \%)$. Les chrétiens représentaient $47,8 \%$ des cas tandis que les musulmans $4,5 \%$.

Le nombre de malades augmentait au fil des années avec une progression temporelle annuelle moyenne d'environ 13,75\%. En 2014, on a enregistré le plus grand nombre de cas représentant $57 \%$ de tous les patients recensés (figure 1).

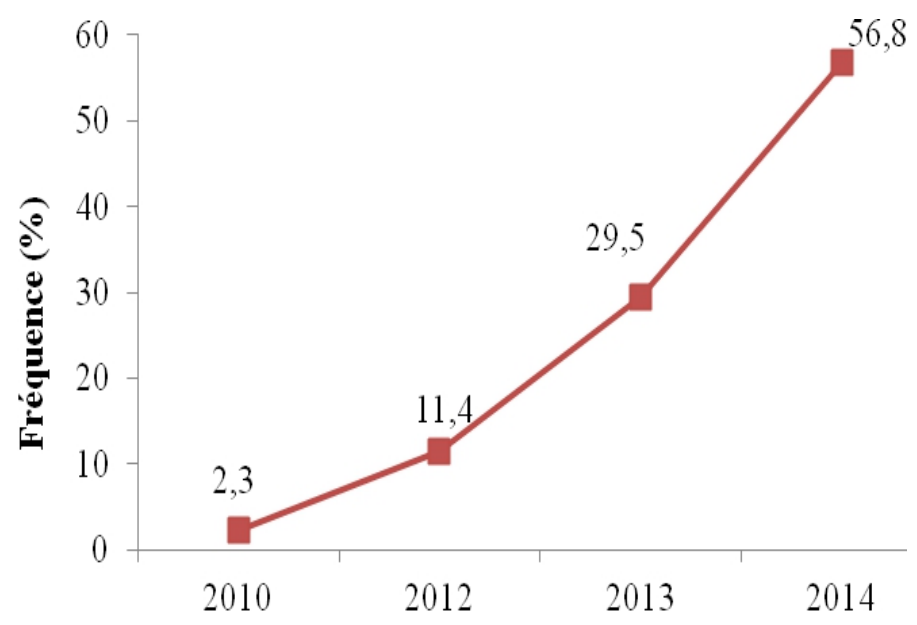

Figure 1 : Répartition temporelle dẹp̣ás de néphropathies médicamenteuses 
L'hypertension artérielle (HTA) était présente chez 50\% des malades. Le diabète et l'alcoolisme étaient présents chez 18,2\% des patients et les hépatites médicamenteuses étaient présentes chez $11,4 \%$ des malades.

La moitié des malades $22(50 \%)$ utilisaient régulièrement les décoctions traditionnelles. Elles étaient suivies de la gentamicine 5(11,4\%), du Paracétamol 4(9,1\%) et du Ceftriazone 3(6,8\%). La pharmacopée traditionnelle $22(50 \%)$ suivie des anti-inflammatoires non stéroïdiens $17(38,6 \%)$ et des antibiotiques 10(22,7\%) étaient respectivement les groupes de drogues les plus utilisées.

L'anurie/oligurie (27,3\%), les œdèmes des membres inférieurs $(27,3 \%)$, les troubles digestifs $(77,3 \%)$, l'asthénie $(59,1 \%)$ et la fièvre $(27,3 \%)$ étaient les principaux signes et symptômes observés.

Les insuffisances rénales aigues étaient présentes chez 65,9\% des malades tandis que les insuffisances rénales chroniques n'étaient présentes que chez $34,1 \%$ des malades. La néphropathie interstitielle et la néphropathie tubulaire étaient les types de néphropathies les plus courants, elles étaient présentes chez $29,5 \%$ des cas. La néphropathie glomérulaire présente chez $15,9 \%$ des malades venaient en deuxième position. La néphropathie fonctionnelle et la néphropathie obstructive présentes respectivement chez $4,5 \%$ et $2,3 \%$ étaient les types les moins présents.

La dialyse a été préconisée chez $59,1 \%$ des malades. Le régime diététique a été prescrit à $52,3 \%$ des malades tandis que la transfusion sanguine a été faite chez $50 \%$ malades. La corticothérapie $(11,4 \%)$ était le traitement le moins administré. Toutefois, il n'y a pas de différence statistiquement significative entre les malades d'IRC sous dialyse et les malades d'IRA sous dialyse. Tous avaient le même risque d'effectuer la dialyse comme traitement.

À l'issu du traitement, $43 \%$ des malades ont guéris contre $14 \%$ de décédés et $20 \%$ étaient encore en cours de traitement pendant l'étude.

\section{Discussion}

La majorité des patients (73\%) était de sexe masculin avec un sexratio de 2,46 en faveur des hommes. Ces résultats sont en accord avec ceux d'Aabach (2012), d'Ahmed (2006) et d'Amellal (2010). Par contre, ils sont en opposition avec ceux d'Izzedine (2012) et Ghafel (2001) qui ont trouvés que les femmes étaient les plus atteintes. Cette différence peut s'expliquer d'une part par le fait que dans les pays en développement, les hommes sont plus enclins aux pétites activités économiques et face à un problème de santé, ils ont plus recours à l'automédication, donc aux «médicaments illicites» qu'aux services de santé. D'autre part, cela peut également s'expliquer par la progression plus rapide des maladies rénales chez l'homme par rapport à la femme. 
L'âge des patients variait entre 11 et 87 ans avec un âge moyen de $48 \pm 18$ ans. Chez Aabach (2012), l'âge moyen des patients était de $53 \pm 2$ ans avec des extrêmes de 15 et 92 ans. En Inde, Avasthi et al. (2003) avaient trouvé une moyenne d'âge de 50,1 ans. En Turquie, Cengiz et al. (2000) avaient trouvé une moyenne d'âge de 54,6 ans. Izzedine et al. (2012), avait trouvé une moyenne d'âge $>50$ ans. Ces résultats sont relativement proches de ceux de notre étude. La moyenne d'âge chez Amellal (2010) était de 40,2 \pm 20.63 ans avec des extrêmes de quatre mois et de 93 ans. Ghafel (2001) a rapporté une moyenne d'âge de $38 \pm 22$ ans. Au Burkina Faso, Lengani (2009) avait rapporté une moyenne d'âge de 38,6 ans. Cette différence peut s'expliquer par le fait que l'âge avancé est un facteur de risque des néphropathies médicamenteuses. En effet, plus on prend de l'âge, plus on consomme des médicaments potentiellement néphrotoxiques ayant un rapport avec les différentes comorbidités (Coresh et al., 2005).

Cependant, les tranches d'âge les plus affectées étaient celles de 45 à 54 ans et de 65 ans plus avec une fréquence de 20,45\% chacune. Aabach (2012), avait noté une prédominance de la tranche de 40 à 80 ans qui représentait $70 \%$ des sujets. Amellal (2010) avait noté le maximum de fréquence dans la tranche d'âge 40-60 ans. Par contre, Ahmed (2006) avait rapporté que $42,9 \%$ des malades étaient de la tranche des 20 à 39 ans. Bourquia (1999) avait relevé que 55\% des malades avaient entre 30 et 40 ans. Toutefois, les néphropathies médicamenteuses touchent aussi bien les vieillards si ceux-ci s'exposent à la consommation anarchique du médicament.

Le secteur informel était le secteur d'activité le plus touché $(27 \%)$ suivis du secteur agricole (20,5\%) et des fonctionnaires (15,9\%). Par contre, les inactifs étaient les moins touchés avec $(6,9 \%)$. Cette fréquence peut s'expliquer par le fait qu'au Cameroun, le marché du travail est caractérisé par une prépondérance du secteur informel (INS, 2005).

Par ailleurs, les plus touchées, avaient des comorbidités d'hypertension artérielle et de diabète. En effet, ces deux pathologies constituent de fait, un facteur de risque des néphropathies. Et donc la prise des médicaments non contrôlés augmente davantage la survenue des atteintes rénales toxiques médicamenteuses chez ces personnes âgées. Aussi, les personnes âgées sont plus vulnérables à la néphrotoxicité, en raison d'une diminution de la fonction rénale et d'une diminution de la capacité de régénération des cellules.

L'hypertension artérielle était présente chez 50\% des patients et le diabète chez $18,2 \%$ des malades. Ces résultats sont en accord avec ceux d'autres études qui classent l'hypertension artérielle, les cardiopathies et le diabète comme facteurs de risque de survenue des néphropathies médicamenteuses. En effet, Aabach (2012), avait trouvé l'hypertension 
artérielle chez $23 \%$ de la population enquêtée et le diabète chez $18,35 \%$. Ahmed (2006), l'hypertension artérielle représentait 52,6\% des comorbidités. Amellal (2010), avait rapporté que $8,8 \%$ des patients étaient hypertendus, $8,8 \%$ souffraient de cardiopathie et $10 \%$ étaient diabétiques. Lengani (2009) avait obtenu $10,7 \%$ des patients ayant une hypertension et $10,7 \%$ de patients ayant une cardiopathie.

La fréquence des néphropathies médicamenteuses au CHUY était de 18,3\%. Ces résultats corroborent ceux de Kleinknecht (1987), Karie (2010) à la suite Nash (2002) et Uchino (2005) qui variaient entre $16 \%$ et $19 \%$. Par contre, ils étaient six fois supérieur à ceux d'Aabach (2012), qui étaient de $3 \%$ et le double de ceux d'Amellal (2010), qui étaient de 9,5\%. Cette fréquence élevée peut s'expliquer par le fait qu'au Cameroun, les médicaments illicites potentiellement néphrotoxiques abondent et l'automédication est très répandue (Ebelle Lottin, 2007). Cette fréquence élevée peut également s'expliquer par les comorbidités hypertension-diabète et l'âge avancé de patients.

La majorité des patients soit $75 \%$ avaient été référés pour hypercréatininémie. Pourtant, les signes et symptômes qui poussaient les malades à consulter étaient constitués des douleurs, des vomissements, de l'asthénie, de l'altération de l'état général et de la conscience. Ces résultats sont sensiblement proches à ceux d'Ahmed (2006), qui rapportaient que $70 \%$ des patients étaient référés pour hypercréatininémie. En effet, l'insuffisance des connaissances des populations sur les néphropathies médicamenteuses, fait en sorte que c'est au cours d'une consultation dans les formations sanitaires qu'ils étaient dépistés et référés.

La fréquence des insuffisances rénales aigues (IRA) était supérieure à celle des insuffisances rénales chroniques (IRC). En effet, les IRA représentaient $65,9 \%$ contre $34,1 \%$ pour les IRC. Ahmed (2006) avait rapporté $87,1 \%$ d'IRC contre 12,9\% d'IRA. Karie (2004), Nortier et al. (2011) ont eu des fréquences d'IRA $<$ à 5\%. Cette différence des résultats du CHUY s'explique par la rapidité de diagnostic et de prise en charge. En effet, une grande partie des patients avait été référée et par conséquent, ils ont pu être diagnostiqués et mis sous traitement à temps avant que la pathologie ne devienne chronique.

Les néphropathies interstitielles et tubulaires (29,5\%) étaient les plus fréquentes suivies des néphropathies glomérulaires $(15,9 \%)$, de néphropathies fonctionnelles $(4,5 \%)$ et des néphropathies obstructives $2,3 \%$. Ces résultats sont en accord avec ceux trouvés par Fillastre et Legallicier (2001), qui avient trouvé que les néphropathies tubulaires étaient comprises entre 8 à 10\%. Aabach (2012), avait retrouvé $17.5 \%$ de néphropathies interstitielles, $5 \%$ de néphropathies glomérulaires, $14,31 \%$ de néphropathies tubulaires aigues, $3,15 \%$ de néphropathies tubulaires et $1,8 \%$ de 
néphropathies vasculaires. Chez Ahmed (2006), les néphropathies vasculaires étaient dominantes soit 44,3\%. Elles étaient suivies des néphropathies glomérulaires $14,3 \%$ et des néphropathies interstitielles 11,4\%. Cette variabilité de la typologie des néphropathies s'explique par la diversité des médicaments mis en cause. En effet, chacun des médicaments incriminés est susceptible d'induire un type spécifique de néphropathie. De plus, la variabilité des antécédents médicaux peut tout aussi expliquer cette diversité de la typologie des néphropathies.

La pharmacopée traditionnelle était incriminée dans la survenue de la moitié des néphropathies (50\%). La gentamicine avec $11,4 \%$ des cas et paracétamol avec $9,1 \%$ étaient respectivement le $2^{\text {ème }}$ et le $3^{\text {ème }}$ médicament mis en cause dans la survenue de ces atteintes. Ce résultat était nettement inférieur à celui d'Amellal (2010) qui avait noté dans sa série l'utilisation de la gentamicine dans $35,7 \%$ des cas.

Les thérapeutiques les plus concourantes dans la survenue des atteintes rénales médicamenteuses provenaient de la pharmacopée traditionnelle $(50 \%)$, les anti-inflammatoires non stéroïdiens $(38,6 \%)$ et des antibiotiques $(22,7 \%)$. Ces résultats sont relativement différents de ceux de Devasmita et Ziauddin (1997), Karie (2005), Matzke (1997) et Nortier (2001) qui avaient trouvé que les classes thérapeutiques les plus fréquemment associés à la survenue des néphropathies médicamenteuses étaient les analgésiques, les antibiotiques, les inhibiteurs de l'enzyme de conversion de l'angiotensine et les produits de contraste. Par contre, les résultats d'Amellal (2010), avaient révélé que les classes médicamenteuses les plus concernées étaient les AINS (21,4\%), les Aminosides (35,7\%), les produits de contraste iodés, certains hydroxyéthylamidons, les diurétiques de l'anse, les inhibiteurs de l'enzyme de conversion (IEC) et les anti-récepteurs de l'angiotensine II (ARAII), les chimiothérapies et l'amphotéricine B. Selon Lord (2002a), les analgésiques étaient responsables de 1 à $3 \%$ des cas d'insuffisance rénale chronique aux États-Unis et la consommation d'AINS augmenterait de $58 \%$ le risque d'insuffisance rénale aiguë. Pour Leven et al. (2014), les antagonistes de la vitamine K (33,3\%), les antibiotiques $(20,8 \%)$, les AINS $(20,8 \%)$ et les IP proton (16,7\%) étaient les classes de médicamenteuses responsables des atteintes médicamenteuses. Cette différence s'expliquer par cette nature traditionaliste des populations camerounaises qui ont recours à l'ethnopharmacopée. De plus, cette variabilité des classes trouve sa justification dans le fait que la «pharmacie de la rue » offre toutes les classes de médicaments. Aussi, la présence des différentes comorbidités joue également un rôle dans la diversification des médicaments et des classes médicamenteuses.

La dialyse était le traitement des atteintes rénales toxiques médicamenteuses le plus préconisé $(59,1 \%$ des cas). Un régime alimentaire 
avait été indiqué chez 52,3\% des malades, la transfusion chez $50 \%$ et la corticothérapie dans $11,4 \%$ des cas. Ces résultats étaient le triple de ceux d'Aabach (2012), qui rapportaient l'hémodialyse chez 18,50\% de la population étudiée. Par contre, ils étaient inférieurs à ceux d'Amellal (2010), qui soulignaient l'hémodialyse chez $69,4 \%$ des cas. Le remplissage vasculaire était indiqué chez 39,5\% des malades, la transfusion chez 39,5\% des malades et la levée d'obstacle dans 14,3\% des cas.

En effet, le traitement administré était fonction des complications présentés par les malades, de la dimension et de la typologie de la néphropathie médicamenteuse. Ce traitement était également fonction du plateau technique présent au CHUY et des moyens financiers des malades. En effet, les transplantions rénales n'étant pas mises en œuvre au Cameroun, les malades dont 1'état nécessitait une transplantation rénale étaient sous hémodialyse à vie.

À l'issu du traitement, $43 \%$ de malade étaient guéris contre $14 \%$ décédés. $20 \%$ des malades étaient en cours de traitement tandis que $7 \%$ étaient sortie contre avis médical. Dans sa série, Aabach (2012) avait rapporté $39 \%$ de guérison complète, $30 \%$ de guérison partielle et $31 \%$ de décès. Amellal (2010) avait obtenu $24 \%$ de guérison complète, $32 \%$ de guérison partielle, $14 \%$ de cas de survenue d'une IRC et $30 \%$ des décès. Cette différence peut être attribuer à la dimension aigue de l'insuffisance rénale dont souffrait la majorité des patients. En effet, le diagnostic précoce et la prise en charge rapide des malades avait permis de limiter la survenue des complications conduisant à la mort ou à des séquelles voire la chronicité.

En somme, le nombre de cas d'atteintes rénales toxiques médicamenteuses au CHU de Yaoundé augmente sensiblement au cours du temps avec une progression temporelle annuelle moyenne d'environ 13,75\%. Cette progression traduit l'ampleur du problème au sein de la population globale. Elle s'explique par la consommation anarchique du médicament par les populations.

\section{Conclusion}

Les atteintes rénales toxiques médicamenteuses comme la plupart des maladies rénales évoluent de façon insidieuse d'où leur diagnostic tardif. Elles restent une préoccupation majeure pour les services de santé. Au Cameroun, les études sur la question restent rares et les populations ignorent les risques et dangers inhérents à la consommation anarchique du médicament. Cette situation s'explique par une insuffisance de sensibilisation des populations sur la consommation incontrôlée du médicament et un laxisme du système de contrôle du médicament. Aussi, par une absence de répression permanente sur la vente illicite du médicament. En effet, 38,6\% des patients de cette étude utilisaient les anti-inflammatoires non stéroïdiens, 22,7\% utilisaient les 
antibiotiques et $50 \%$ utlisaient les médicaments de la pharmacopée traditionnelle. La lutte contre ce fléau passe par la mise en œuvre d'un plan de sensibilisation des populations sur les dangers de la consommation anarchique des médicaments et le renforcement de la pharmacovigilance.

\section{References:}

Aabach, A.(2012). Profil epidemiologique de l'insuffisance Renele aigue intrahospitaliere. Thèse de Doctorat en Médecine. Université Sidi Mohammed Ben Abdellah, Faculté de Médecine et de Pharmacie. Fes, Maroc.

Ahmed, M. A. (2006). Problematique de la prise en charge des insuffisants renaux chroniques en dialyse a l'hopital du point G en 2005. Thèse de Doctorat en Médecine. Université de Bamako, Faculté de Médecine, de Pharmacie et d'Odontostomatologie. Bamako, Mali.

Amellal, R. (2010). Activité de l'unité de réanimation néphrologique en 2008-CHU IBN ROCHD. Thèse de Doctorat en Médecine. Université Sidi Mohammed Ben Abdellah, Faculté de Médecine et de Pharmacie. Fes, Maroc.

Article L.5111-1 du Code de la Santé Publique en France modifié par la loi n²007-248 du 26 février 2007-art.3 JORF du 27 février 2007. Consulté sur le site web www.sante.gouv.fr le 13 septembre 2015.

Avasthi G. et al. (2003). Acute Renal Failure in Medical and Surgical Intensive Care Units-A One Year Prospective Study. Renal failure. 25(1): 105-113.

Bourquia, A. (1999). État actuel du traitement de l'insuffisance rénale chronique au Maroc. Néphrologie. 20.

Cengiz, U. et al. (2000). Acute renal failure in central Anatolia. NDT, 15 : 152-155.

Claude, B. (1872). Leçons de pathologie expérimentale.

Coresh, J. et al., (2005). Chronic kidney disease aw areness, prevalence and trends among U.S. adults, 1999 to 2000. J Am Soc Nephrol. 16:180-8.

Dahl, N.V. (2001). Herbs and supplements in dialysis patients: panacea or poison? Seminar in dialysis, 14 (3):186-92.

Devasmita, C. et Ziauddin, A. (1997). Drug-induced nephrotoxicity. Med Clin North Am. 81 (3): 705-17.

Ebelle Lottin, M.F. (2007). Etude des hépatites médicamenteuses dans les services de gastroentérologie de l'hôpital central et du CHU de Yaounde (Cameroun). Thèse de Doctorat en Pharmacie. Université de Bamako, Faculté de Médecine, de Pharmacie et d'Odonto-Stomatologie. Bamako, Mali.

Eva, F. M. (2013). Revue de la littérature sur la Toxicité et les effets indésirables médicamenteux des antipaludiques. Thèse de Doctorat en 
Pharmacie. Université des Sciences, des techniques et des technologies. Bamako, Mali.

Fondjo, P. (2009). Contrefaçon de médicaments, mortel poison. Repéré le 15-02-2015 à http://www.youtube.co/watch?v=OHHhwJoNao. Cotonou, Bénin.

Ghafel, C., Niang, A., Benghanem, G. M., Hachim, K., Ramdani, B. \& Zaïd, D. (2001). Facteurs pronostiques de l'insuffisance rénale aiguë. Néphrologie. $22(5): 236$.

Institut National de la Statistique. (2005). Enquête sur l'Emploi et le Secteur Informel (EESI) - Phase 1: Enquête sur l'Emploi. Rapport Principal. Yaoundé-Cameroun.

Izzedine, H., Deroy, G. et Salpêtrière, P. (2012). Insuffisances rénales aigues et Néphrotoxiques. Séminaire national de Néphrologie.

Karie, S., Launay-Vacher, V. et Deray, G. (2005). Néphrotoxicité des médicaments : veille bibliographique janvier 2003-décembre 2004. Néphrologie \& Thérapeutique. 285-295.

Karie, S., Launay-Vacher, V. ; Deray, G., Isnard-Bagnis C. (2010). Toxicité rénale des médicaments. Néphrologie \& Thérapeutique. 6 :58-74.

Larouche, J. et Berner, M. (2001). Les interactions entre les médicaments sous ordonnance et les produits naturels. L'Actualité pharmaceutique. p. 9 (10) : 30-1.

Lengani, A. D., Kargougou, G.B., Fogazzi, M. L. (2009). L’insuffisance rénale aigue au Burkina Faso. Néphrol ther.

Leven, C. et al. (2014). Prospective study of drug-induced interstitial nephritis in eleven French nephrology units. Presse Med. 43(11) : 369-76.

Lord, A. et Ménard, C. (2002a, juin). La néphrotoxicité médicamenteuse : comment limiter les dégâts ? Le Médecin du Québec. 37(6).

Matzke, G.R. et Frye, R.F. (1997). Drug administration in patients with renal insufficiency: minimizing renal and extra renal toxicity. Drug Safety. 16 (3) : 205-31.

Mbassi-Bikele, Y. (2014). Médicament de la rue : un tueur silencieux. Récupéré le 15-02-2015 sur Cameroun Tribune. : https://www.cameroontribune.cm/index.php?option=com_content $\&$ view $=$ article $\&$ id $=81174 \% 3 \mathrm{Ame}$ dicament-de-la-rue-un-tueur-silencieux \&catid=3\% Adossier-de-la-redaction Nortier, J., Depierreux, M. et Vanherweghem, J-L. (1999). Phytothérapie et néphrotoxicité. Rev Med Brux. 1 : 9-14.

Nortier, J., Hougardy, J.- M., Pozdzik, A., Gastaldello, K. et LeMoine, A. (2011). Insuffisance (atteinte) rénale aiguë et néphrotoxicité médicamenteuse. Rev Med Brux. 32 : 305-11.

OMS (2004). Les comités pharmaceutiques et thérapeutiques. Guide pratique. Geneva : OMS. 
Tanguy, B. P. (2011). Le tueur silencieux. [Film Documentaire]. Paris : L'Harmatan.

WHO (2008). Medicines: safety of medicines - adverse drug reactions. Fact sheet. N²93. In: WHO. Geneva. 